\title{
Variational principles and the shift in the front speed due to a cutoff
}

\author{
Vicenç Méndez, ${ }^{1}$ Daniel Campos, ${ }^{2}$ and Evgeny P. Zemskov ${ }^{2}$ \\ ${ }^{1}$ Departament de Medicina, Facultat de Ciències de la Salut, Universitat Internacional de Catalunya, c/ Josep Trueta s/n, \\ E-08190 Sant Cugat del Vallès (Barcelona) Spain \\ ${ }^{2}$ Grup de Física Estadística, Departament de Física, Universitat Autònoma de Barcelona, E-08193 Bellaterra (Barcelona) Spain
}

(Received 11 April 2005; published 10 November 2005)

\begin{abstract}
We apply variational principles to reaction-diffusion equations in order to predict, for a general reaction term, the sign of the shift in the front speed due to a cutoff. We develop an improved variational principle to obtain the shift in the front speed for a wide range of reaction terms, and the theoretical results so obtained are in excellent agreement with numerical solutions. This work proves that variational principles are an optimal framework to deal with fronts propagating into unstable and metastable states under cutoff.
\end{abstract}

DOI: 10.1103/PhysRevE.72.056113

PACS number(s): 05.70.Ln, 05.40.Fb

\section{INTRODUCTION}

Variational principles have been developed to find bounds to the speed of reaction-diffusion fronts [1]. They allow one to obtain lower bounds for the front speed when the linear speed is selected and when the marginal stability analysis fails. The variational principles can thus be applied to general reaction terms and, in particular, to pulled and pushed fronts propagating into unstable or metastable states [1]. A pulled front is one that propagates into an unstable state with an asymptotic speed equal to the linear speed; that is, the computed speed from the linear analysis around the unstable state. A front that propagates into an unstable state whose asymptotic speed is higher than the linear speed is referred to as a pushed front [2]. As applications of variational principles, we can mention the study of front speed in hyperbolic reaction-diffusion equations $[3,4]$, thermal combustion waves [5], reaction-convection-diffusion equations [6], nonlocal reaction-diffusion equations [7], reaction with anomalous diffusion [8-10], and other nonlinear dynamical systems $[11,12]$. This method becomes really useful when one is able to obtain lower and upper bounds that coincide: the front speed is then known precisely. However, this is frequently a challenging task and one has to resign oneself to dealing with bounds.

On the other hand, the concept of a cutoff has been considered to model the effect of the discreteness of particles. Brunet and Derrida [13] have studied fronts propagating into unstable states, and they showed that the shift on the speed has the form $-\pi^{2}(\ln \varepsilon)^{-2}$, showing a strong dependence on the cutoff threshold $\varepsilon$. A little bit later, Kessler et al. [14] discussed the problem of the time development of leading edges, precursors, and cutoffs in fronts propagating into metastable states, finding a power-law dependence for the speed shift on the cutoff threshold. In this work, we want to apply variational principles to determine, for a wide variety of reaction terms, the lower bound for the front speed for the reaction-diffusion equation

$$
\partial_{t} u=\partial_{x x} u+f(u),
$$

where the reaction function $f(u)$ contains a cutoff. First, we start by showing that variational principles maydefine the sign of the shift in terms of the analytic properties of the reaction function. In particular, we show that when the cutoff is considered on the unstable state then the shift is negative and it reduces the front speed. However, when it is considered on the metastable state, then the shift is positive and it increases the front speed. We propose a variational principle, more adequate to study the cutoff effect, and we show that it improves the previous variational principles and also provides lower bounds with an excellent agreement with numerical solutions. In consequence, this work shows that variational principles are a general framework able to describe itself the cutoff effect on the speed of fronts propagating into unstable and metastable states.

\section{VARIATIONAL PRINCIPLE: POSITIVE AND NEGATIVE SHIFTS}

We consider monotonically decreasing fronts $u=u(z=x$ $-c t)$ propagating with constant asymptotic speed $c$ joining $u=1$ and $u=0$. The front satisfies $u^{\prime \prime}+c u^{\prime}+f(u)=0$, under the boundary conditions $u(z \rightarrow \infty)=0$ and $u(z \rightarrow-\infty)=1$, where the prime symbol stands for derivative respect to $z$. From the usual variational principle, the asymptotic front speed is given by [1]

$$
c^{2}=\max _{g \in S}\left[2 \frac{\int_{0}^{1} f g d u}{\int_{0}^{1}\left(g^{2} / h\right) d u}\right],
$$

where $h=-d g / d u$ and $S$ is the set of positive and monotonically decreasing trial functions $g(u)$ in $(0,1)$ for which the integrals involved in Eq. (2) are convergent. On frequent occasions it is not possible to know the form of the function $g=g(u)$ which maximizes the relationship in brackets in Eq. (2) and one can find only lower bounds 


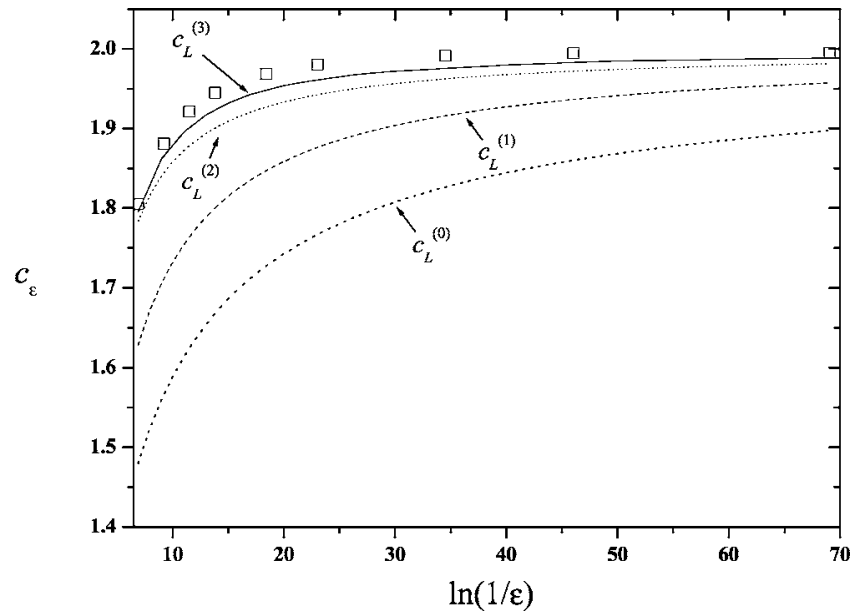

FIG. 1. Front speed (dimensionless) versus the cutoff for pulled fronts propagating into unstable states. Symbols (squares) correspond to numerical solutions obtained from the integration of Eq. (1) with $f(u)=\theta(u-\varepsilon) u(1-u)$. Curves corresponds to the solutions obtained from the variational principles. In particular, $c_{L}^{(0)}$ is obtained from the old variational principle $(10), c_{L}^{(1)}$ is obtained from the new variational principle (11) by using $g_{\alpha}^{(0)}(u), c_{L}^{(2)}$ is obtained from the improved variational principle by using $g_{\alpha}^{(1)}(u)$ and $c_{L}^{(3)}$ is obtained from the biparametric family $g_{\alpha, \beta}^{(2)}(u)$ and the improved variational principle.

$$
c_{L}^{2}=2 \frac{\int_{0}^{1} f g d u}{\int_{0}^{1}\left(g^{2} / h\right) d u}
$$

To consider a cutoff in the reaction function we can write $f(u)=\theta(u-\varepsilon) \varphi(u)$, where $\varepsilon$ is the cutoff threshold and $\varphi(u)$ may be a positive or negative function in $(0,1)$. Then,

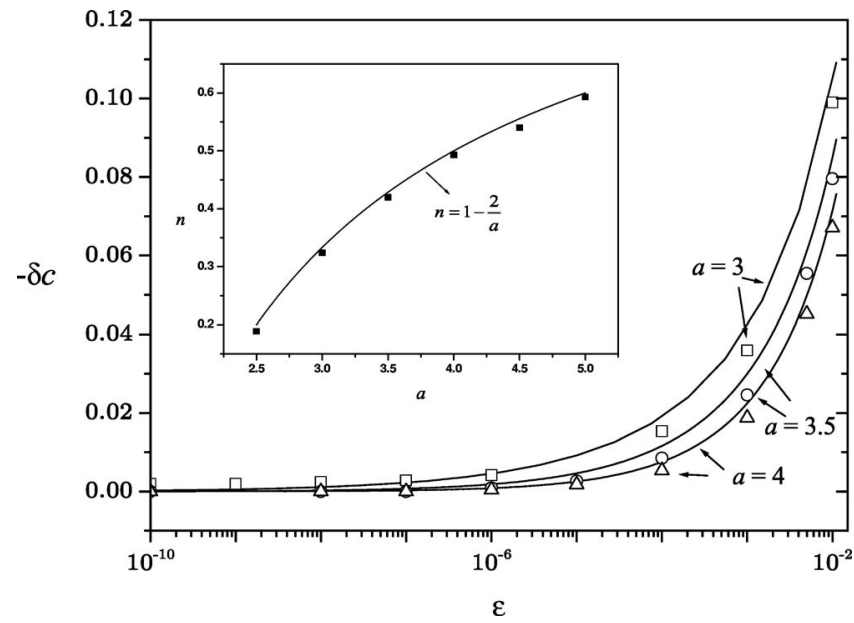

FIG. 2. Front speed (dimensionless) versus the cutoff for the GL case. Solid lines correspond to the results obtained from Eq. (13) and symbols are the numerical solutions computed from (1) with $f(u)=\theta(u-\varepsilon) u(1-u)(1+a u)$. In particular, we take $a=3(\square)$, $a=3.5(\circ)$, and $a=4(\triangle)$. Inset: symbols ( $\square$ ) are obtained by fitting our results to a power law in $\varepsilon$ and solid curve is the exponent calculated below Eq. (13).

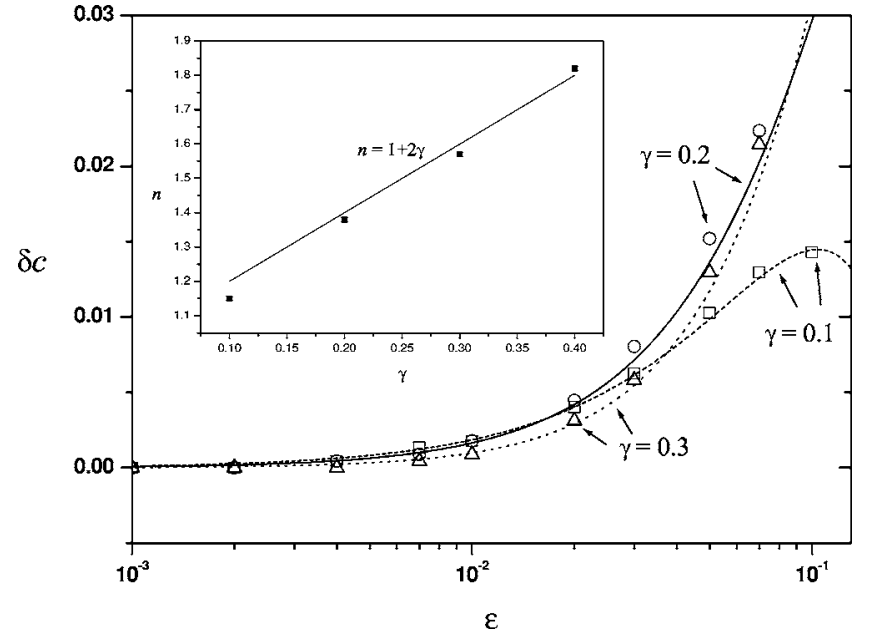

FIG. 3. Front speed (dimensionless) versus the cutoff for the bistable case. Solid lines correspond to the results obtained from Eq. (14) and symbols are the numerical solutions computed from (1) with $f(u)=\theta(u-\varepsilon) u(1-u)(u-\gamma)$. In particular, we take $\gamma=0.1($ $\square$, dashed line), $\gamma=0.2(\circ)$, solid line), and $\gamma=0.3$ ( $\triangle$, dotted line). Inset: symbols ( $\mathbf{\square})$ are obtained by fitting our results to a power law in $\varepsilon$, and the solid curve is the exponent calculated below Eq. (14).

$$
\int_{0}^{1} f g d u=\int_{\varepsilon}^{1} \varphi g d u=\int_{0}^{1} \varphi g d u-\int_{0}^{\varepsilon} \varphi g d u .
$$

If $\varphi(u)>0$ for $u \in(0, \varepsilon]$ with $0<\varepsilon<1$, then $\int_{0}^{\varepsilon} \varphi g d u>0$ and, from (4), the inequality $\int_{0}^{1} f g d u<\int_{0}^{1} \varphi g d u$ always holds. Hence, from Eq. (2) one obtains

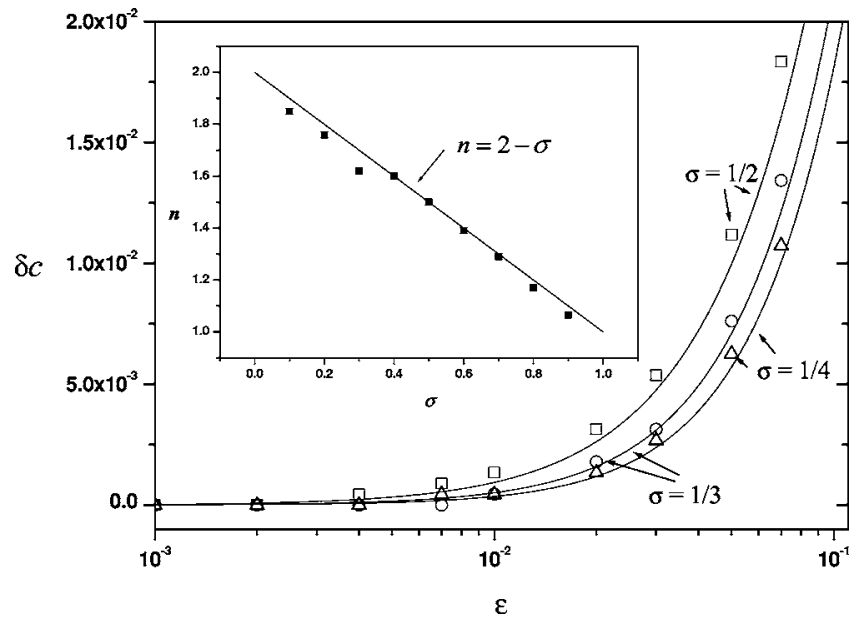

FIG. 4. Front speed (dimensionless) versus the cutoff for the Schlögl reaction function. Solid lines correspond to the results obtained from Eq. (15) and symbols are the numerical solutions computed from (1) with $f(u)=\theta(u+1-\varepsilon)\left(1-u^{2}\right)(u+\sigma)$. In particular, we take $\sigma=1 / 4(\triangle), \sigma=1 / 3(\circ)$ and $\sigma=1 / 2(\square)$. Inset: symbols (ם) are obtained by fitting our results to a power law in $\varepsilon$, and the solid curve is the exponent calculated below Eq. (15). 


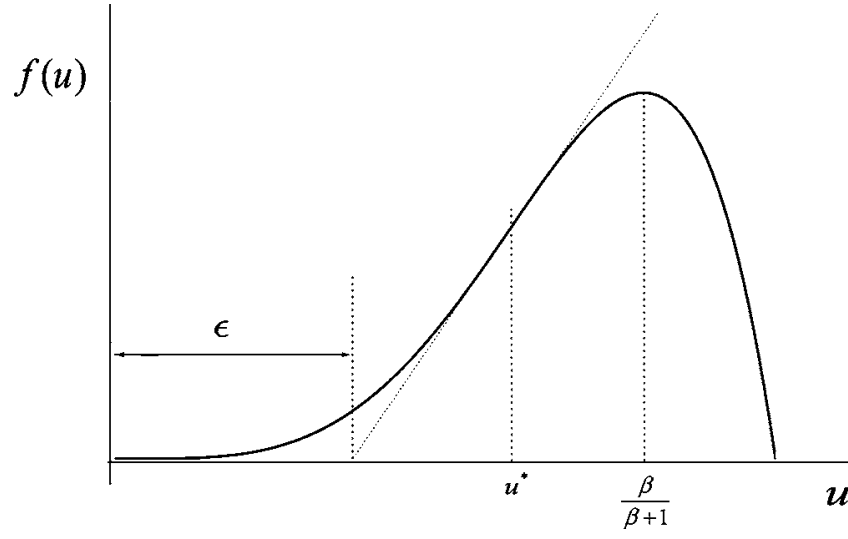

FIG. 5. Schematic plot of the combustion reaction term $f(u)=u^{\beta}(1-u)$. The inflection point $u^{*}$ defines a tangent line which cross the horizontal axis at $\epsilon$ which represents a intrinsic cutoff.

$$
c_{\varepsilon}^{2}=2 \max _{g \in S}\left[\frac{\int_{0}^{1} f g d u}{\int_{0}^{1}\left(g^{2} / h\right) d u}\right]<2 \max \left[\frac{\int_{0}^{1} \varphi g d u}{\int_{0}^{1}\left(g^{2} / h\right) d u}\right]=c_{0}^{2} .
$$

Defining $c_{\varepsilon}=c_{0}+\delta c$, from (5), the shift $\delta c$ is negative and the presence of a cutoff reduces the front speed. However, if the cutoff is such that $\varphi(u)<0$ for $u \in(0, \varepsilon]$ with $0<\varepsilon<1$, then $\int_{0}^{\varepsilon} \varphi g d u<0$ and the shift $\delta c$ is positive: the presence of a cutoff increases the front speed. For example, in the case $f(u)=\theta(u-\varepsilon) u(1-u)$ one has $\delta c<0$, while for $f(u)=\theta(u-\varepsilon) u(1-u)(u-b)$ with $0<\varepsilon<b$, one has $\delta c>0$. Thus, we have shown how variational principles are able to infer the sign of the shift in the front speed due to a cutoff in a very general way.

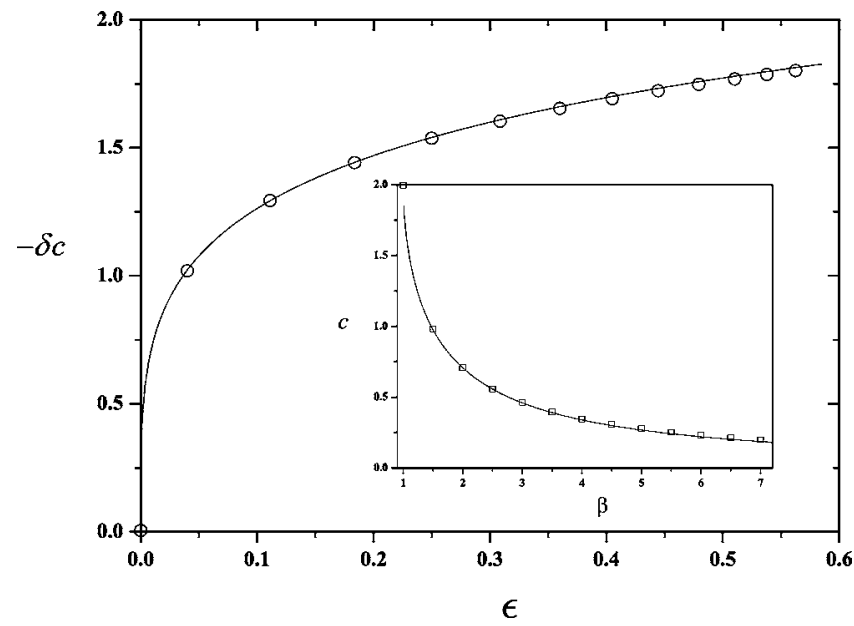

FIG. 6. Front speed for combustion fronts (dimensionless) versus the intrinsic cutoff $\epsilon$. In the inset, the front speed versus the exponent $\beta$ is plotted. Solid curves stand for theoretical results obtained from Eq. (16) and symbols $(\circ)$ are numerical solutions of (1) with $f(u)=u^{\beta}(1-u)$.

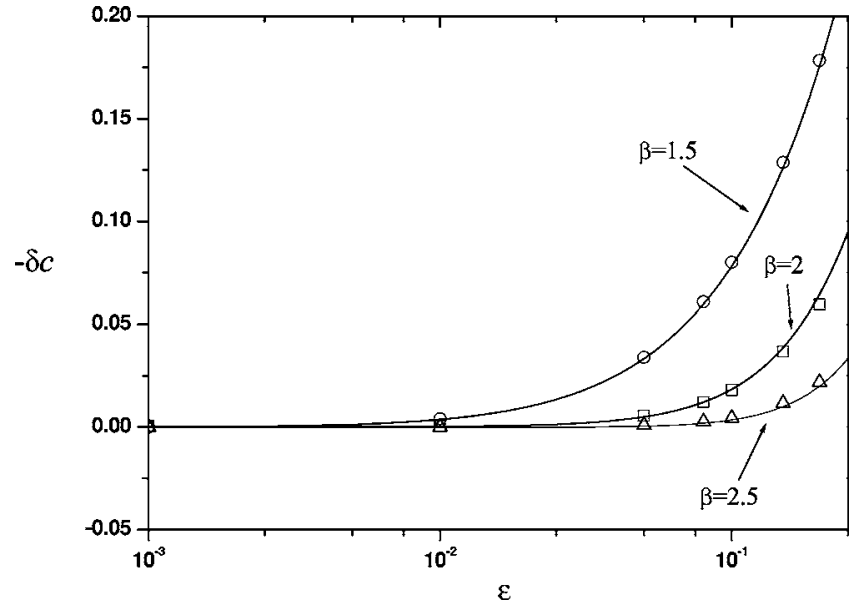

FIG. 7. Front speed (dimensionless) versus the cutoff for combustion case with external cutoff. Solid lines correspond to the results obtained from Eq. (17) and symbols are the numerical solutions computed from (1) with $f(u)=\theta(u+1-\varepsilon)\left(1-u^{2}\right)(u+\sigma)$. In particular, we take $\beta=1.5(\circ, \epsilon=0.04), \beta=2(\square, \epsilon=0.111)$, and $\beta=2.5(\triangle, \epsilon=0.184)$.

\section{IMPROVED VARIATIONAL PRINCIPLE}

We split the equation $u^{\prime \prime}+c u^{\prime}+f(u)=0$ in two regions: region I, where $0<u<\varepsilon$ and region II, where $\varepsilon<u<1$. Defining $p_{\mathrm{I}}=-d u_{\mathrm{I}} / d z$, and $p_{\mathrm{II}}=-d u_{\mathrm{II}} / d z$, one has the following differential equations for front

$$
\begin{gathered}
p_{\mathrm{I}} \frac{d p_{\mathrm{I}}}{d u_{\mathrm{I}}}-c_{\varepsilon} p_{\mathrm{I}}=0, \\
p_{\mathrm{II}} \frac{d p_{\mathrm{II}}}{d u_{\mathrm{II}}}-c_{\varepsilon} p_{\mathrm{II}}+\varphi\left(u_{\mathrm{II}}\right)=0 .
\end{gathered}
$$

From the first equation in (6) one finds $p_{\mathrm{I}}=c u_{\mathrm{I}}$, and multiplying the second one by $g$ and integrating over $u_{\mathrm{II}}$, we have

$$
-\frac{1}{2} g(\varepsilon) \varepsilon^{2} c_{\varepsilon}^{2}+\int_{\varepsilon}^{1} g \varphi\left(u_{\mathrm{II}}\right) d u_{\mathrm{II}}=\int_{\varepsilon}^{1} d u_{\mathrm{II}}\left(c_{\varepsilon} g p_{\mathrm{II}}-\frac{1}{2} h p_{\mathrm{II}}^{2}\right),
$$

where we have made use of the matching condition for the front slope at $u=\varepsilon$; that is, $p_{\mathrm{II}}(\varepsilon)=p_{\mathrm{I}}(\varepsilon)=c_{\varepsilon} \varepsilon$ in the integration by parts of $\int_{\varepsilon}^{1} d u_{\mathrm{II}} g p_{\mathrm{II}} d p_{\mathrm{II}} / d u_{\mathrm{II}}$. Since $c_{\varepsilon}, p_{\mathrm{II}}, g$, and $h$ are positive for fixed $u_{\mathrm{II}}$, the function $\Phi\left(p_{\mathrm{II}}\right) \equiv c_{\varepsilon} g p_{\mathrm{II}}-1 / 2 h p_{\mathrm{II}}^{2}$ has a maximum at $p_{\mathrm{II}}^{\max }=c_{\varepsilon} g / h$, so that $\Phi\left(p_{\mathrm{II}}\right) \leqslant c_{\varepsilon}^{2} g^{2} /(2 h)$ at each value of $u_{\mathrm{II}}$. It then follows that

$$
c_{\varepsilon}^{2} \geqslant c_{L}^{2}(\varepsilon)=\frac{2 \int_{\varepsilon}^{1} g \varphi d u}{\int_{\varepsilon}^{1}\left(g^{2} / h\right) d u+\varepsilon^{2} g(\varepsilon)} .
$$

This lower bound is valid for any reaction function for which a monotonic front exists. To prove that this is a variational principle we have to show that there exists a function $g$ for which this equality holds. From $p_{\mathrm{II}}^{\max }=c_{\varepsilon} g / h$, one can see that the equality is attained when $\hat{g}$ is such that 
$\hat{g}^{\prime} / \hat{g}=-c_{\varepsilon} / p_{\mathrm{II}}$, which may be integrated to obtain

$$
\hat{g}\left(u_{\mathrm{II}}\right)=\exp \left(-c_{\varepsilon} \int_{u_{0}}^{u_{\mathrm{II}}} d u_{\mathrm{II}} / p_{\mathrm{II}}\right)
$$

with $\varepsilon<u_{0}<1$.

Since $p_{\mathrm{II}}$ vanishes at $u_{\mathrm{II}}=1$, we must analyze the behavior of $\hat{g}$ at this point in order to ensure the convergence of the integrals in (7). Near $u_{\mathrm{II}}=1$ one has $p_{\mathrm{II}} \sim r\left(1-u_{\mathrm{II}}\right)$ to the lowest order, where $r$ is the positive root of $r^{2}+r c_{\varepsilon}-\varphi^{\prime}(1)=0$. Thus, from Eq. (8) we obtain $\hat{g} \sim(1$ $\left.-u_{\mathrm{II}}\right)^{r / c_{\varepsilon}} ; \hat{g} \varphi$ and $\hat{g}^{2} / h$ diverge at most as $\left(1-u_{\mathrm{II}}\right)^{r / c_{\varepsilon}+1}$, and the integrals in (7) exist. Therefore, the asymptotic front speed is given by

$$
c_{\varepsilon}^{2}=\max _{g \in S}\left[\frac{2 \int_{\varepsilon}^{1} g \varphi d u}{\int_{\varepsilon}^{1}\left(g^{2} / h\right) d u+\varepsilon^{2} g(\varepsilon)}\right],
$$

where the maximum is taken over all positive decreasing functions $g$ in $(0,1)$ for which the integrals exist. Note that in the case $\varepsilon=0$, Eq. (9) reduces to Eq. (2). Note that the variational principle in Eq. (9) is valid in general for any reaction term. As the problem of searching the trial function $g$, which maximizes the expression in brackets in Eq. (9) and transforms the inequality in Eq. (7) into an equality, is a rather difficult task, we deal with finding the best lower bound, and it will be compared to numerical solution directly performed on the reaction-diffusion equation $\partial_{t} u=\partial_{x x} u+\theta(u-\varepsilon) \varphi(u)$ under a steep initial condition. This shall be done in turn for fronts propagating into unstable and metastable states.

\section{FRONTS PROPAGATING INTO UNSTABLE STATES}

We consider here two special cases: the KolmogorovPetrovskii-Piskunov (quadratic) and the Ginzburg-Landau (GL) (cubic) reaction functions. In both cases we will compare our lower bound with numerical solutions and with the results of Brunet and Derrida [1] and Kessler et al. [14].

\section{A. Pulled fronts propagating into unstable states}

First we consider the family of trial functions $g_{\alpha}^{(0)}(u)=u^{\alpha-1}$ as in [1]. Taking $\varphi(u)=u(1-u)$, from the old variation principle [Eq. (3)] the best lower bound is

$$
c_{L}^{(0)}(\varepsilon)=\left[2 \max _{-1<\alpha<1}\left(1-\alpha^{2}\right)\left(\frac{1-\varepsilon^{\alpha+1}}{1+\alpha}-\frac{1-\varepsilon^{\alpha+2}}{2+\alpha}\right)\right]^{1 / 2} .
$$

Second, we consider the same family $g_{\alpha}^{(0)}(u)$, but using now the variational principle developed in Sec. III, we find

$$
\begin{aligned}
c_{L}^{(1)}(\varepsilon) & =\left[2 \max _{-1<\alpha<1} \frac{1-\alpha}{2+\alpha} \frac{1+(1+\alpha) \varepsilon^{2+\alpha}-(2+\alpha) \varepsilon^{\alpha+1}}{1-\alpha^{2} \varepsilon^{\alpha+1}}\right]^{1 / 2} \\
& =2 \sqrt{\frac{\ln \varepsilon-\varepsilon+1}{\ln \varepsilon-2}} \simeq 2-\frac{3}{\ln (1 / \varepsilon)}+\frac{15}{4} \frac{1}{(\ln \varepsilon)^{2}} .
\end{aligned}
$$

Finally, as the aim here is to compare the improvement of the new variational principle, we consider alternatively the family $g_{\alpha}^{(1)}(u)=[(1-u) / u]^{1-\alpha}$ also in the new variational principle to obtain

$$
c_{L}^{(2)}(\varepsilon)=\left[2 \max _{-1<\alpha<1}(1-\alpha) \frac{\Gamma(1+\alpha) \Gamma(3-\alpha)-6 G_{1}(\alpha, \varepsilon)}{\Gamma(1+\alpha) \Gamma(3-\alpha)-6 G_{1}(\alpha, \varepsilon)+6(1-\alpha) \varepsilon^{1+\alpha}(1-\varepsilon)^{1-\alpha}}\right]^{1 / 2} \simeq 2-\frac{5}{4 \ln (1 / \varepsilon)}-\frac{323}{192} \frac{1}{(\ln \varepsilon)^{2}}
$$

where $G_{1}(\alpha, \varepsilon) \equiv \varepsilon^{1+\alpha}{ }_{2} F_{1}(\alpha-2, \alpha+1 ; 2+\alpha, \varepsilon) /(1+\alpha)$ and ${ }_{2} F_{1}$ is the hypergeometric function. In the last equality of Eq. (12) we have used the fact that the maximum is attained very close to $\alpha=-1$, as may be checked numerically by plotting (12) versus $\alpha$ for some values of $\varepsilon$. Our goal here is to compare (11) with (12) in order to find which family of trial functions fits better with numerical solutions. In Fig. 1 we compare $c_{L}^{(0)}, c_{L}^{(1)}$ and $c_{L}^{(2)}$ (dashed lines) with numerical solutions (symbols). Two interesting results may be found: first of all, the new variational principle improves the old one, and secondly, the agreement with the family $g_{\alpha}^{(1)}(u)$ is better than with $g_{\alpha}^{(0)}(u)$. However, the best fit is not in an excellent agreement with numerical solutions or with the Brunet and Derrida's result $c_{\varepsilon}=2-\pi^{2}(\ln \varepsilon)^{-2}$ [13]. To improve the result of $c_{L}^{(2)}$, we try with the biparametric family of trial functions $g_{\alpha, \beta}^{(2)}(u)=u^{\alpha-1}(1-u)^{\beta}$. In this case, the new lower bound $c_{L}^{(3)}$ involves integrals that have to be numerically performed as well as the maximum over $\alpha$ and $\beta$. When $\varepsilon \rightarrow 0$, the maximum is attained at large $\beta$ and $\alpha \rightarrow 0$. For $\varepsilon \sim 1$, it is attained at $\beta \rightarrow 0$ and $\alpha \rightarrow 1$. In Fig. 1, the lower bound is depicted in solid line and exhibits good agreement with the numerical solutions. 


\section{B. Pushed and pulled fronts propagating into unstable states}

We consider here a front connecting the unstable state $u$ $=0$ to the stable state $u=1$ of the GL reaction function $\varphi(u)=u(1-u)(1+a u)$ with $a>0$. First of all, we have to check that when $\varepsilon=0$, our result is in agreement with that obtained by Ben-Jacob et al. [15]. Making use of the family $g_{\alpha}^{(1)}(u)$, we get

$$
c_{L}(0)=\left\{2 \max _{-1<\alpha<1}(1-\alpha)\left[1+\frac{a}{4}(1+\alpha)\right]\right\}^{1 / 2} .
$$

When $a<2$, the maximum is reached at $\alpha=-1$ and $c_{L}(0)=2$ (pulled front), while for $a>2$, the maximum is attained at $\alpha=-2 / a$ and $c_{L}(0)=\sqrt{2 / a}+\sqrt{a / 2}$ (pushed front) in agreement with [15].

Now, we consider $\varepsilon \neq 0$ and $g_{\alpha}^{(1)}(u)$. In this case, from (7) we get

$$
c_{L}^{2}(\varepsilon)=2 \max _{-1<\alpha<1}(1-\alpha) \frac{\Gamma(1+\alpha) \Gamma(3-\alpha)-6 G_{1}(\alpha, \varepsilon)+\frac{a}{4} \Gamma(2+\alpha) \Gamma(3-\alpha)-6 a G_{2}(\alpha, \varepsilon)}{\Gamma(1+\alpha) \Gamma(3-\alpha)-6 G_{1}(\alpha, \varepsilon)+6(1-\alpha) \varepsilon^{1+\alpha}(1-\varepsilon)^{1-\alpha}},
$$

where $G_{2}(\alpha, \varepsilon) \equiv \varepsilon^{2+\alpha}{ }_{2} F_{1}(\alpha-2, \alpha+2 ; 3+\alpha, \varepsilon) /(2+\alpha)$. Taking the first derivative respect to $\alpha$ in Eq. (13) and performing a perturbative expansion in powers of $\varepsilon$, it is easy to show that the maximum is reached at $\alpha \simeq-2 / a+$ (higher-order terms). On setting this into (13), $c_{L}^{2}(\varepsilon) \sim O(0)+\varepsilon^{1-2 / a}$ and $\delta c \sim \varepsilon^{1-2 / a}$ to the leading order, in agreement with the result already obtained by Kessler et al. [14]. In Fig. 2, we compare the lower bound obtained from (13) with our numerical integrations and with the scaling relation $\delta c \sim \varepsilon^{1-2 / a}$ (inset). An excellent agreement is found. The symbols in the inset of Fig. 2 correspond to find the exponent $n$ after fitting the results for $\delta c$ obtained from (13) to a power law $\varepsilon^{n}$. It is shown here that the shift $\delta c$ is negative and the cutoff reduces the front speed, in agreement with our results in Sec. II.

\section{FRONTS PROPAGATING INTO METASTABLE STATES}

In this section we deal with front propagation into the metastable state. We consider here the bistable reaction term employed in front propagation in excitable media [16] and the Ginzburg-Landau cubic reaction functions. As in the previous section, we will compare the results derived from the variational principle with numerical solutions and the Kessler's scaling relations.

\section{A. Bistable reaction function}

Let us consider a front joining the metastable state $u=0$ to the stable state $u=1$ for the reaction function $\varphi(u)=u(1-u)(u-\gamma)$ with $0<\gamma<1 / 2$. Taking the family $g_{\alpha}^{(1)}(u)$ one obtains, for $\varepsilon=0$,

$$
c_{L}(0)=\left\{2 \max _{-1<\alpha<1}(1-\alpha)\left[\frac{1+\alpha}{4}-\gamma\right]\right\}^{1 / 2}=\frac{1}{\sqrt{2}}-a \sqrt{2},
$$

in agreement with the already known result [16]. For $\varepsilon \neq 0$ we find from $(7)$

$$
c_{L}^{2}(\varepsilon)=\frac{1}{2} \max _{-1<\alpha<1}(1-\alpha) \frac{\Gamma(2+\alpha) \Gamma(3-\alpha)-24 G_{2}(\alpha, \varepsilon)-4 a \Gamma(1+\alpha) \Gamma(3-\alpha)+24 a G_{1}(\alpha, \varepsilon)}{\Gamma(1+\alpha) \Gamma(3-\alpha)-6 G_{1}(\alpha, \varepsilon)+6(1-\alpha) \varepsilon^{1+\alpha}(1-\varepsilon)^{1-\alpha}} .
$$

Following the steps of the previous section, we obtain here that the maximum is attained at $\alpha \simeq 2 \gamma+$ (higher-order terms). Thus, from (14), $\delta c \sim \varepsilon^{1+2 \gamma}$ to the leading order. This case was not dealt by Kessler et al., but the scaling law may be also obtained following his estimations. In Fig. 3 we plot in curves the results obtained from (14) which are in very good agreement with numerical solutions (symbols) and with the scaling law (inset). It is worthwhile to mention that here the shift $\delta c$ is positive, in agreement with the general result of Sec. II. Thus, the cutoff here increases the front speed, contrary to what happens for pulled fronts propagating into unstable states.

\section{B. Schlögl reaction function}

We deal with the Schlögl reaction function to study the front propagation into the metastable state. To do this, we consider the case studied by Kessler et al. [14], in which $f(u)=\theta(u+1-\varepsilon)\left(1-u^{2}\right)(u+\sigma)$ with $0<\sigma<1$. We consider a front connecting the metastable state $u=-1$ to the stable state 
$u=1$. In order to apply the variational principle, we have to define a new field $\rho$ such that $\rho(z \rightarrow \infty)=0$ and $\rho(z \rightarrow-\infty)$ $=1$. If we define $\rho=(1+u) / 2$, and introduce it into the general reaction-diffusion equation, we obtain $\rho^{\prime \prime}+c \rho^{\prime}+4 \theta(\rho$ $-\varepsilon / 2) \rho(1-\rho)[\rho-(1-\sigma) / 2]=0$. Computing the front speed without a cutoff $(\varepsilon=0)$ with the family $g_{\alpha}^{(1)}(u)$ we get, after some algebra,

$$
\begin{aligned}
c_{L}(0) & =\left\{2 \max _{-1<\alpha<1} \frac{1-\alpha}{2-\alpha}[(1+\alpha)(1-\sigma-\alpha)-3(1-\sigma)]\right\}^{1 / 2} \\
& =\sigma \sqrt{2},
\end{aligned}
$$

which is the expected result [16]. When cutoff is present, from (7), the lower speed reads

$$
c_{L}^{2}(\varepsilon)=2 \max _{-1<\alpha<1}(1-\alpha) \frac{\Gamma(2+\alpha) \Gamma(3-\alpha)[1+\alpha-2(1-\sigma)]-24 G_{2}(\alpha, \varepsilon / 2)+12(1-\sigma) G_{1}(\alpha, \varepsilon / 2)}{\Gamma(1+\alpha) \Gamma(3-\alpha)-6 G_{1}(\alpha, \varepsilon / 2)+6(1-\alpha)(\varepsilon / 2)^{1+\alpha}(1-\varepsilon / 2)^{1-\alpha}} .
$$

The maximum is then reached at $\alpha=1-\sigma+$ (higher-order terms) and then the shift scales as $\delta c \sim \varepsilon^{2-\sigma}$ to the leading order, in agreement with Kessler's results. In Fig. 4 we show that the agreement is excellent between variational results, numerical solutions, and the power-law scaling (inset). In this case, there is also a positive shift $\delta c$ on the speed.

\section{INTERNAL CUTOFF}

Up to now, we have considered the cutoff as a consequence of the discrete nature of the particles which has to be introduced into our equation by hand. Now we want to point out that some reaction functions incorporate their own cutoff. For example, combustion reaction functions take into account the existence of an ignition threshold (by means of an activation energy or an ignition temperature) such that for values of the field lower than the threshold, there is no reaction. Another example may be the interaction between green and burning trees in a forest fire model [17]. If $\beta$ stands for the number of burning trees needed to set fire to a neighboring green tree, then $f(u)=u^{\beta}(1-u)$. $\beta$ plays the role of an internal cutoff because in the neighborhood of the state $u=0$, the reaction term is very small and there exists a plateau region such that for a finite range $f \simeq 0$. In Fig. 5, we draw $f(u)=u^{\beta}(1-u)$ qualitatively to illustrate this behavior. If we call $u^{*}$ the horizontal coordinate of the inflection point, it is easy to find that $u^{*}=(\beta-1) /(\beta+1)$. Tracing the tangent line to $f$ at the inflection point, a cutoff $\epsilon$ may be arbitrarily defined as the distance between 0 and the crossing point between the tangent line and the horizontal axis; i.e., $\epsilon$ $=[(\beta-1) /(\beta+1)]^{2}$.

To obtain the lower bound to the front speed, we take the family $g_{\alpha}^{(1)}(u)$ and obtain from Eq. (3)

$$
c_{L}^{2}(\beta)=\frac{12}{\Gamma(3+\beta)} \frac{\left(1-\alpha^{*}\right) \Gamma\left(\alpha^{*}+\beta\right)}{\Gamma\left(1+\alpha^{*}\right)},
$$

where $\alpha^{*}$ is solution of the equation $\Psi\left(\alpha^{*}+\beta\right)-\Psi\left(\alpha^{*}+1\right)$ $=\left(1-\alpha^{*}\right)^{-1}$ and $\Psi(x) \equiv d \ln \Gamma(x) / d x$. As may be seen in Fig. 6 , there is an excellent agreement between the front speed computed from (16) (solid lines) and the numerical solutions (symbols). We have fitted $\delta c=c_{L}(\beta)-c_{L}(\beta=1)$ to a power law and we have obtained $\delta c=-2.39 \epsilon^{0.26}$. Thus, in combustion reaction terms, the cutoff $\epsilon$ acts qualitatively, as in a GL reaction function when the front propagates into the unstable state.

\section{Internal and external cutoff}

We consider now an external cutoff on the previous combustion reaction term; that is, $f(u)=\theta(u-\varepsilon) u^{\beta}(1-u)$. It is expected that if $\varepsilon \ll \epsilon$ then the effect of the external cutoff is insignificant because it cuts a region where $f \simeq 0$. To appreciate the effect of the external cutoff, we will need to deal with high values of $\varepsilon$. From Eq. (7) and also assuming the family $g_{\alpha}^{(1)}(u)$, one gets for this case

$$
c_{L}^{2}(\varepsilon, \beta)=2 \max _{-\beta<\alpha<1}(1-\alpha) \frac{\frac{\Gamma(\beta+\alpha) \Gamma(3-\alpha)}{\Gamma(3+\beta)}-G_{3}(\alpha, \beta, \varepsilon)}{\frac{\Gamma(1+\alpha) \Gamma(3-\alpha)}{\Gamma(4)}-G_{1}(\alpha, \varepsilon)+(1-\alpha) \varepsilon^{1+\alpha}(1-\varepsilon)^{1-\alpha}},
$$


where $\quad G_{3}(\alpha, \beta, \varepsilon) \equiv \varepsilon^{\beta+\alpha}{ }_{2} F_{1}(\alpha-2, \alpha+\beta ; \beta+\alpha+1, \varepsilon) /(\beta$ $+\alpha$ ). In Fig. 7 we observe really good agreement between numerical solutions (symbols) and the results obtained from (17) (solid lines) for different values of $\beta$. It is observed that appreciable shifts occur for $\varepsilon \sim 10^{-1}$, a very high value for a cutoff.

In this case, the shift is negative, as in the case of front propagation into unstable states. Likewise, it is interesting to see that $u=0$ has an indefinite stability because $\varphi^{\prime}(0)=0$. Finally, we note that when $\beta=2$, it is possible to find an exact solution for the maximum in Eq. (17) because it is attained at $\alpha=1$, and then

$$
c_{L}(\varepsilon, 2)=\frac{1}{\sqrt{2}}(1-\varepsilon) \sqrt{\frac{1+3 \varepsilon}{1+\varepsilon+\varepsilon^{2}}} \simeq \frac{1}{\sqrt{2}}-\frac{3}{\sqrt{2}} \varepsilon^{2}+\cdots .
$$

\section{CONCLUSIONS}

In this work we have applied variational principles to study the effect of a cutoff on the speed of propagating reaction-diffusion fronts. We have shown, in a general way, that variational principles determine the sign of the shift in the front speed induced by a cutoff threshold. If $f(u)=\theta(u$ $-\varepsilon) \varphi(u)$, where $\varepsilon$ stands for the cutoff, it is shown that the sign of the shift depends on the sign of the reaction function $\varphi(u)$ in the interval $(0, \varepsilon]$. The shift is positive if $\varphi(u)_{u \in(0, \varepsilon]}<0$ (the front propagates into the metastable state) and it is negative if $\varphi(u)_{u \in(0, \varepsilon]}>0$ (the front propagates into the unstable state).

We have also improved the variational principle to deal with reaction-diffusion with a cutoff for fronts propagating into unstable and metastable states. Our first application has been devoted to pulled fronts propagating into unstable states, where we have proved that the new lower bound for the front speed improves the results obtained from the old variational principle, widely employed in the literature. However, to get a better agreement with numerical solutions and the Brunet and Derrida's result we have considered a biparametric family of trial functions. The family $g_{\alpha}^{(1)}(u)$ $=[(1-u) / u]^{1-\alpha}$ has been considered to obtain lower bounds for pushed fronts propagating into unstable and metastable states. The theoretical and numerical results are in excellent agreement and the results obtained by Kessler et al. have been analytically recovered.

We have discussed the existence of an internal cutoff in combustion reaction terms and the external inclusion of an additional cutoff. We have obtained lower bounds, in very good agreement with numerical solutions. Variational principles are shown specially interesting for dealing with combustion fronts with a cutoff because the methods of neither Brunet-Derrida nor Kessler et al. are valid to calculate the shift. In this case, it is not possible to obtain the exact solution for the front speed when $\varepsilon=0$, nor is it possible to calculate front solutions near the state $u=0$ by linearizing the traveling wave equation due to the nonlinearities of the reaction term.

\section{ACKNOWLEDGMENTS}

E.P.Z. thanks Professor J. Casas-Vázquez and his group for warm hospitality in Barcelona. This work has been supported by the MCYT under Grant No. BFM 2003-06033.
[1] R. D. Benguria and M. C. Depassier, Phys. Rev. Lett. 77, 1171 (1996).

[2] U. Ebert and W. van Saarloos, Physica D 146, 1 (2000); W. van Saarloos, Phys. Rep. 386, 29 (2003).

[3] V. Méndez, J. Fort, and J. Farjas, Phys. Rev. E 60, 5231 (1999).

[4] R. D. Benguria and M. C. Depassier, Phys. Rev. E 66, 026607 (2002).

[5] R. D. Benguria, J. Cisternas, and M. C. Depassier, Phys. Rev. E 52, 4410 (1995).

[6] R. D. Benguria, M. C. Depassier, and V. Méndez, Phys. Rev. E 69, 031106 (2004).

[7] R. D. Benguria and M. C. Depassier, Phys. Rev. E 57, 6493 (1998).

[8] R. D. Benguria and M. C. Depassier, Phys. Rev. E 52, 3285 (1995).
[9] V. Méndez and J. Fort, Phys. Rev. E 64, 011105 (2001).

[10] J. Fort and V. Méndez, J. Stat. Phys. 107, 805 (2002).

[11] R. D. Benguria and M. C. Depassier, Phys. Rev. Lett. 77, 4482 (1996); R. D. Benguria and M. C. Depassier, Phys. Rev. Lett. 77, 2847 (1996); R. D. Benguria and M. C. Depassier, Phys. Rev. E 59, 4889 (1999).

[12] V. Méndez, J. Math. Phys. 39, 954 (1998).

[13] E. Brunet and B. Derrida, Phys. Rev. E 56, 2597 (1997).

[14] D. A. Kessler, Z. Ner, and L. M. Sander, Phys. Rev. E 58, 107 (1998).

[15] E. Ben-Jacob, I. Brand, G. Dee, L. Kramer, and J. S. Langer, Physica D 14, 348 (1985).

[16] J. D. Murray, Mathematical Biology, 3rd ed. (Springer-Verlag, Berlin, 2003).

[17] V. Méndez and J. E. Llebot, Phys. Rev. E 56, 6557 (1997). 